\title{
Micro physical simulation system of electric power systems
}

\author{
Xin Xu, Zongshuai Jin, Hengxu Zhang* \\ Key Laboratory of Power System Intelligent Dispatch and Control of Ministry of Education (Shandong University), Jinan 250061, \\ P.R. China
}

\begin{abstract}
Micro physical simulation system is a new open type micro physical simulation system of electric power system, which is simple structure, small-scale, low cost, flexible installation and is an open system including generation, transmission, transformation, load and synchronous monitoring systems. It is a small-scale dynamic simulation system based on similarity theory, which has the same physical properties as the actual electric power system and can be used in electrical engineering-related research and education.
\end{abstract}

Keywords: Power system, dynamic simulation, micro, wide-area monitoring system (WAMS)

\section{Introduction}

Dynamic simulation of electric power system is a very important way to analyze the dynamic characteristics and the physical laws of the electric power system, and to test relay protection devices and secondary equipment. It is also an effective and efficient method in the education of electrical engineering, to give the students an overview of the electric power system. However, the traditional dynamic simulation laboratory, which uses large-scale physical elements, needs huge investment, large experimental area, high operation cost and high maintenance cost, which obstructs the popularization of dynamic simulation as a tool in research and education [1]. The digital simulation, like RTDS, brings all the elements into a flexible experiment platform, which leads to low cost and small area. But the algorithms in the these devices are based on the laws that are already known, which means that it can be hardly used to discover the features of the new elements in the power systems. Additionally, because of its structure like a "black box", it has obvious limit that the students cannot get an overview of the electric power system without the existence of physical elements, which weakens its value in education.

To solve these problems, a new open type micro physical simulation system of electric power system is introduced in this paper, which is simple structure, low cost, flexible installation, and is an open type system includes micro simulated generation, transmission, transformation, load and synchronous monitoring systems.

\section{Detailed Description of Micro Physical Simulation System of Electric Power Systems}

\subsection{Overall introduction}

Micro physical simulation system of electric power system contains all the modules in the actual system, including micro generation, transmission, transformation, load, and synchronous monitoring systems. Micro generation module contains the simulations of prime motor, generator, AGC (Automatic Generation Control) and AVC (Automatic Voltage Control) in traditional thermal power generation and hydroelectric power generation. It also contains the simulations of renewable energy generation and nuclear power generation. Micro transformation module contains the simulations of transformers with

\footnotetext{
* Manuscript received June 10, 2014; revised July 18, 2014.

Corresponding author: Hengxu Zhang; Tel.: +86-531-88392838; E-mail address: zhanghx@ sdu.edu.cn

doi: $10.12720 /$ sgce.4.1.72-76
} 
different types and voltage levels. Micro transmission module contains the simulation s of impedance and admittance of the transmission lines. Micro load module contains resistive loads, asynchronous machine and so on. Micro synchronous monitoring module contains the simulation of WAMS.

\subsection{Micro generation module}

Micro generation module contains the simulations of prime motor, generator, AGC and AVC in traditional thermal power generation and hydroelectric power generation. It also contains the simulations of renewable energy generation and nuclear power generation. The whole construction of micro generation module is shown in Fig. 1. The device is shown in Fig. 2.

The prime motor is simulated by DC servo motor, which is powered by rectified power source and smoothing reactor [2]. The rotational speed of the motor is proportional to the output DC voltage $U_{c}$ of the rectified power source, which can be controlled by the AGC controller. The generator is simulated by small-scale three-phase synchronous generator. When generating, the generator is driven by the motor through a coaxial shaft. Specifically, the exciting field of the generator is established by the controllable DC exciter, which uses the adjustable switching stabilized power supply and can be controlled by the AVC controller.

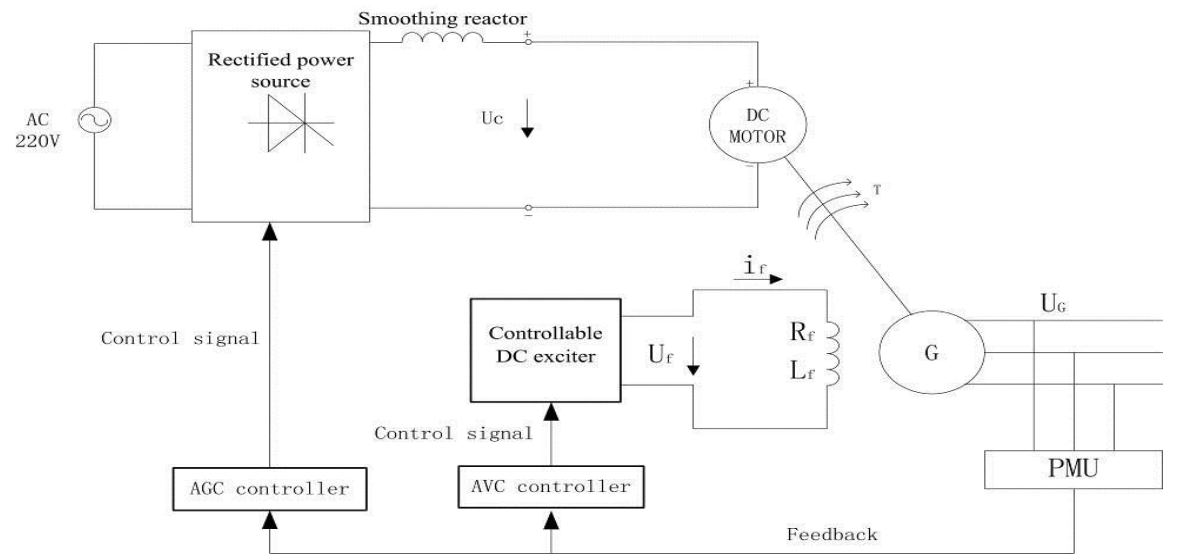

Fig. 1. Micro generation module construction.

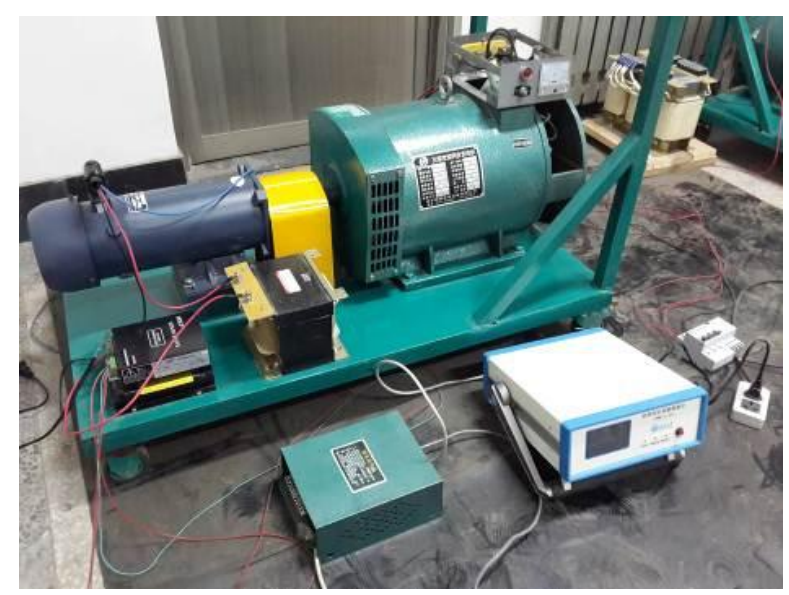

Fig. 2. Micro generation device.

AGC and AVC are both negative feedback system and generally consist of several parts: the measurement of voltage, current, frequency and power; the feedback and data processing; the controller. The measurement part uses the PMU (Phase Measurement Unit), designed by Key Laboratory of Power System Intelligent Dispatch and Control of Ministry of Education in Shandong University. It can measure 
the amplitude, phase angle, frequency of the voltage and the current, and the magnitude of the power [3], as shown in Fig. 3. The feedback part is realized by serial communication, which brings the data from PMU to the controller. The core of the controller can be DSP or ARM, which can handle the complex program and the massive data very well. With proper algorithm routine, the controller can process the original data and export the control signal to hold the amplitude and frequency of the voltages in the stable range.

\section{Fig. 3. PMU device.}

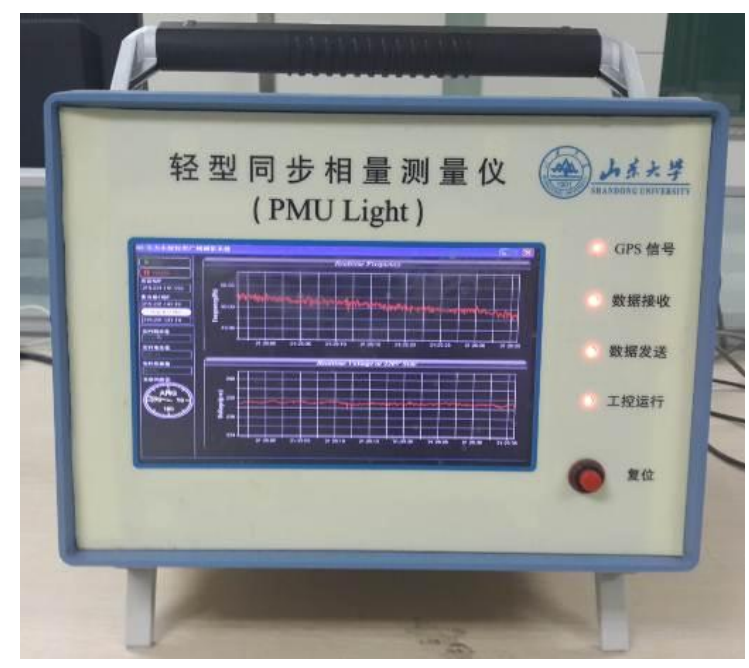

The working process of AGC and AVC is shown in Fig. 1. AGC mainly controls the voltage frequency of the generator [4]. By collecting the frequency data of $U_{G}$ from PMU and calculating, the control signal array is generated from the controller and applied to the rectified power source, which decided the magnitude of the output DC voltage that controlled the rotational speed of the DC motor. AVC mainly controls the voltage amplitude. By getting RMS voltage data of $U_{G}$ from PMU and calculating, the AGC controller forms the control signal array which is then applied to the controllable DC exciter to control the exciting voltage $U_{f}$ of the generator.

The core analyzing algorithm of both the AGC and AVC controller is open type, which means it can be changed according to different requirements or test new control methods.

\subsection{Micro transmission module}

Micro transmission module contains lumped parameter model, $\pi$ type equivalent circuit, which ignores the distribution characteristics of the line and shows the parameters of line in distributive form [5]. These parameters are serial resistance, serial inductance, parallel conductance and parallel susceptance. These four parameters all use adjustable elements, to simulate the lines with different length and distributive parameters. Moreover, all of the physical elements use standardizing elements, whose minimum adjustable step is standardized in order to build the grid easily and compute relevant unknown quantities easily.

\subsection{Micro transformation module}

Micro transformation module also uses standardizing elements. The transformers are all single-phase type, and three transformers form a three-phase transformer [6]. Unusually a group of transformers can simulate one or several transformers in actual electric power system. To make sure that the p.u. of the transformers is the same as the prototype, the compensation should be made by using series impedance and parallel admittance. The mode of connection and magnetic circuit system should be the same with the prototype as well. Because of different neutral grounding mode, the zero sequence impedance $\mathrm{X} \%$ should be compensated on the neutral point of the transformer by using series impedance. 


\subsection{Micro load module}

Micro load module can simulate the active power and passive power absorbed by the actual power load, which shows the effect of the amplitude and frequency change of the bus voltage to the dynamic behavior of the load power. There are two schemes for load simulation: first, the traditional model includes asynchronous motor load model, resistance load model and serial-parallel capacitance load model [7]; second, the controllable load model based on the power electronic equipment. Both of the schemes can simulate the true character of the actual load. The traditional load model contains asynchronous motor load model, resistance load model and serial-parallel capacitance load model. The load simulation is realized according to the actual power load construction, by using the serial-parallel connection of the real electric consuming devices. The controllable load model, based on the power electronic equipment, is a versatile quality controllable load disturbance source, which can effectively simulate the load characters including harmonics, passive power, active power, current, various load characteristics and three-phase current imbalance. The advantages of controllable load model can effective overcome the weakness in traditional load model, like small-scale, less elements, low cost, simple online change, and high simulating accuracy. This kind of model can realize multi-goal complex simulation, and the energy can be returned to the power grid to achieve energy saving.

\subsection{Micro synchronous monitoring module}

The micro synchronous monitoring system has two parts: dynamic monitor system and dynamic control system. The dynamic monitor system is similar to the WAMS in the actual system. This part is mainly realized by the PMU that described in the micro generation module. When experimenting, the PMUs can be easily placed in designated area to gather the data and send to the server or the host computer. The dynamic control system contains system start and stop, voltage level setting, frequency setting, generator automatic parallel, automatic low frequency decrease load control, automatic voltage control, automatic load control and so on. Through the control command from the host computer, these functions are implemented by the cooperation of the primary equipment, such as the circuit-breaker, and the secondary devices such as the AGC and AVC controller in the micro generation module. The control program in the host can easily be changed due to different requirements.

\subsection{Similarity theory}

The similarity theory can be described as follows: the impedance and admittance p.u. value of the generator, transmission line and transformer are the same as the prototype.

To make the p.u. value of the transformers the same as the prototype, $P_{K}, P_{0}$ and $I_{0} \%$ should be close to the prototype and $U_{K} \%$ must be the same with the prototype. Series impedance and parallel admittance are also needed to compensate the parameters, too.

To satisfy the requirement of similarity theory, both the type and the parameters of the generator should be similar to the prototype generator. Then adjust it by changing the capacity slightly. The lack of leakage reactance $X_{d}$ can be compensated by increasing the leakage reactance of the transformer or using series impedance. Mechanical inertia time constant $T_{i}$ can be adjusted by flywheel. Electromagnetic effect inertia time constant $T_{d 0}$ can be adjusted by side reactance device.

\section{Conclusion}

In this paper, a new open type micro physical simulation of electric power systems is introduced. The system is simple structure, small-scale, low cost, flexible installation, and contains all the components in the electric power system includes the generation, transmission, transformation, load and monitor. It can be built as an innovative experiment platform for the electric power system research. It is very useful in researching the problem that cannot be accurately described by math equations. The accuracy of current theory and education method in electric technology can also be tested by this system in order to improve the theory and the education methods. 


\section{Acknowledgements}

This work was supported by the National High Technology Research and Development Program of China (863 Program, No.2011AA05A118).

\section{References}

[1] Kuffel R, Giesbrecht J, Maguire T, Wierckx RP, McLaren P. RTDS-A fully digital power system simulator operating in real time. In: Proc. WESCANEX 95. Communications, Power, and Computing. Conference Proceedings., IEEE, 1995; 2:300-305.

[2] Pillay P, Krishnan R. Modeling, Simulation and analysis of permanent-magnet motor drives. II. The brushless DC motor drive. Industry Applications, 1989; 25(2):274-279.

[3] Widergren SE, Huang Z, Dagle JE. Electric system-wide measurements: North american directions. In: Proc. System Sciences, 2007. HICSS 2007. 40th Annual Hawaii International Conference, 2007:120-120.

[4] H-S Park, K-J Kim. A study on AGC scheme based on real time frequency characteristics. In: Proc. Universities Power Engineering Conference, 2008. UPEC 2008. 43rd International, 2008:1-5.

[5] Saadat H. Power System Analysis. 2nd ed. Singapore: WCB/McGraw-Hill; 1999.

[6] Chapman S. Electric Machinery Fundamentals. 4nd ed. McGraw-Hill Companies; 2003.

[7] System Dynamic Performance Subcommittee Power System Engineering Committee. Standard load models for power flow and dynamic performance simulation. IEEE Transactions on Power Systems, 1995; 10(3):1302-1313. 